Gen Relativ Gravit (2011) 43:337-358

DOI 10.1007/s10714-010-1114-1

GOLDEN OLDIE

\title{
Republication of: \\ Observations in cosmology
}

\author{
Jerome Kristian • Rainer K. Sachs
}

Published online: 13 November 2010

(C) The Author(s) 2010. This article is published with open access at Springerlink.com

An editorial note to this paper and a biography can be found in this issue preceding this Golden Oldie and online via doi:10.1007/s10714-010-1112-3.

Original paper: Astrophysical Journal 143, 379-399 (1966). Reprinted with the kind permission of the American Astronomical Society and of R. K. Sachs. J. Kristian died before the paper was selected for the Golden Oldies.

Editorial responsibility: A. Krasiński, e-mail: akr@camk.edu.pl.

J. Kristian (Deceased June 22, 1996)

Madison, WI, USA

R. K. Sachs $(\varangle)$

Department of Mathematics, University of California, 970 Evans Hall, \# 3840,

Berkeley, CA 94720, USA

e-mail: sachs@math.berkeley.edu 


\title{
OBSERVATIONS IN COSMOLOGY
}

\author{
J. KRISTIAN* AND R. K. SACHS \\ Relativity Center, The University of Texas, Austin, Texas \\ Received May 7, 1965
}

\begin{abstract}
Observations of cosmological effects in anisotropic, inhomogeneous cosmological models are discussed in detail, with numerical estimates. The first and last sections of the paper form a self-contained unit for readers who are unfamiliar with Riemannian geometry. The other sections contain mathematical derivations.

Three assumptions are made: (i) that the universe is described by a Riemannian space time with slowly varying metric tensor; (ii) that light travels along null geodesics and obeys the usual area-intensity law; and (iii) that the gravitational field is related to the matter by Einstein's field equations for dust. The third assumption is not needed for many of the results; the second assumption is proved in the geometric optics limit, assuming a general relativistic model.

The importance of trying to observe angular variations in the various cosmological effects is emphasized. It is shown that otherwise unobservable anisotropies or inhomogeneities can easily give the observed order of magnitude and either sign for the acceleration parameter measured in any one direction via redshifts. A model-independent law for the apparent area of a distant object is given. Detailed equations for number counts and for apparent proper motions are given. It is pointed out that observations to date do not exclude the possible presence of anisotropies and inhomogeneities whose dynamical effects are comparable to the dynamical effects of the expansion.

A new effect, the "distortion effect," is discussed. In any anisotropic model, all distant objects in a particular direction on the celestial sphere may appear distorted, with a definite preferential direction for their longest dimension. The effect gives a direct measurement of space-time curvature and may be observable. But a positive result of the measurement would not favor general relativity over other theories in which assumptions (i) and (ii) above hold.
\end{abstract}

\section{INTRODUCTION}

In cosmology, the universe is usually assumed spatially isotropic or at least homogeneous, except for local irregularities whose distance scale is too small to have cosmological significance (Heckmann and Schücking 1959, 1962; McVittie 1956, 1959, 1962; for inhomogeneous models see Bondi 1947; Hoyle and Narlikar 1961). The observational basis of this assumption is dubious, however, in view of the scarcity of observations near the galactic plane and recent evidence pointing to possible superclustering of galaxies. Therefore, it seems reasonable to ask what observational consequences anisotropies or largescale inhomogeneities would have. In this paper we attempt to answer this question systematically. We are mainly interested in inhomogeneities whose scale is something like $10^{9}$ light-years or more, with smaller scale variations smoothed out as before, though we shall also briefly discuss the possibility that fluctuations whose scale is several orders of magnitude smaller might have measurable effects which would give information on the curvature of space-time. A detailed presentation of our results, with all technical details suppressed and with a Newtonian notation, is given in $\S$ III.2.

Inhomogeneities and anisotropies have not been much discussed in the literature because finding a global cosmological model which does not have a high degree of symmetry is very difficult. But a global model, with boundary conditions and everything else included, is far more than we need to discuss most of the observations that might be made in the near future (see, e.g., Sandage 1961). If we concentrate on those features of any model that have the most direct observational significance, then a very general treatment can be given using standard mathematical techniques.

We make three fundamental assumptions in this paper. In order of importance, these

* Present address: Washburn Observatory, University of Wisconsin, Madison, Wisconsin. 
are the following. (i) The geometry of space time is Riemannian, and all quantities of interest can be expanded in a power series around here-and-now as origin. This assumption amounts to saying that the distance we can see into the universe is small compared both with some reasonably defined radius of curvature and with the scale of the inhomogeneities which remain when local fluctuations are smoothed out. (ii) Light beams travel along null geodesics and obey the intensity versus area law discussed in $\S$ II.1. We shall prove this second assumption in the geometric optics limit for general-relativistic models; in other models, the assumption has usually been made ad hoc. (iii) The metric is governed by the Einstein field equations for dust. This third assumption is not used in deriving the general expressions of $\S$ II. But to get numerical estimates of certain effects we need some dynamical model, and we have chosen the relativistic dust model as an illustration because it is simple and currently popular. Except for these three assumptions our treatment is general; it is rigorous except for the approximations mentioned in them.

The reader interested in the conceptual framework or mathematical structure of general relativity will find nothing in this paper that is not at least implicit in the literature. We are merely concerned with applying the theory to a specific problem.

In § II, we use assumptions (i) and (ii), but not (iii), to derive expressions for five observational effects in general Riemannian space-times. These effects are given as power series, the coefficients of which describe the distribution of matter and the structure of space-time in our vicinity. In $\S \mathrm{III}$, we specialize these results to general-relativistic dust models, writing the equations for observable effects in three-plus-one dimensional notation, and we make some crude numerical estimates. The reader who has no knowledge of general relativity should go directly to $\$$ III.2, which is intended to be comprehensible to anyone who has read § I, knows Cartesian (i.e., Euclidean) tensors, and is willing to take the results on faith.

Three of the results discussed in $\S$ III. 2 deserve special mention. (a) In all cosmological models (general relativistic or not) there is a universal relation

$$
d A=r^{2} d \Omega .
$$

(R. Penrose [private communication] has since proved by a method independent of power series that this relation is exact.) Here $d A$ is the intrinsic cross-sectional area of a distant object; $r$ is a measured quantity, the "corrected luminosity distance," defined by equation (19); and $d \Omega$ is the measured solid angle subtended by the distant object. (b) Measurements of the redshift or other cosmological effects in only one direction should be interpreted with extreme caution. Only knowledge of how an effect varies over the celestial sphere gives really decisive information on the structure of the universe. (c) There is one effect, the "distortion effect," which deserves investigation. It turns out that, in general, we see an intrinsically spherical distant object as an ellipse on our plates. The effect, if it exists and can be measured, gives a simple, direct measurement of spacetime curvature. Absorption corrections are not needed to detect the effect, and theories of galactic evolution are not needed to interpret it. It is similar to the gravitational lens effect, but is caused by the cosmological curvature rather than that of a single large body.

In $\S$ III the "incident electric-type gravitational field" $E_{\alpha \beta}$ plays an important role. We now give a simple Newtonian analogue to this quantity. Let $\phi(x, t)$ be the Newtonian gravitational potential. We set $\phi(0,0)=0$ and expand $\phi(x, 0)$ in a power series about the origin. The gradient $\left(\phi_{, a}\right)_{0}$ is zero in a freely falling frame. ${ }^{1}$ Thus the first non-zero coefficients in the power series are the six quantities

$$
(\phi, a \beta)_{0} \text {. }
$$

\footnotetext{
${ }^{1}$ Here and throughout, Greek letters range and sum from 1 to 3 , Latin letters from 1 to 4 , commas denote ordinary derivatives, semicolons denote covariant derivatives, and a subscript " 0 " on a quantity means that the quantity is to be evaluated at $x=0, t=0$ after all indicated differentiations have been performed.
} 
Now the trace, $\nabla^{2} \phi$, of this Cartesian tensor is directly determined by the local matter density $\rho_{0}$ via Poisson's equation. But the trace-free part,

$$
E_{\alpha \beta}=(\phi, a \beta)_{0}-\frac{1}{3} \delta_{\alpha \beta} \nabla^{2} \phi_{0},
$$

where $\delta_{a \beta}$ is the Kronecker delta, is independent of $\rho_{0}$ and really arises from the over-all lopsidedness of the matter distribution or of the boundary conditions. For example, $E_{a \beta}=0$ if the origin is chosen at the center of a spherically symmetric mass distribution, but $E_{a \beta} \neq 0$ if the origin is chosen at any off-center point. We call $E_{a \beta}$ the incident electric-type gravitational field and $\nabla^{2} \phi_{0}$ the "locally determined" gravitational field.

The same kind of splitting can be carried out for the higher-order coefficients in the expansion of $\phi(x, 0)$. We introduce a standard notation: round and square brackets around indices denote complete symmetrization and antisymmetrization, respectively; for example,

$$
\begin{aligned}
& A_{(\alpha \beta \gamma)}=\frac{1}{6}\left(A_{a \beta \gamma}+A_{\gamma \alpha \beta}+A_{\beta \gamma a}+A_{\beta a \gamma}+A_{\gamma \beta a}+A_{a \gamma \beta}\right), \\
& A_{[a \beta \gamma]}=\frac{1}{6}\left(A_{a \beta \gamma}+A_{\gamma \alpha \beta}+A_{\beta \gamma a}-A_{\beta a \gamma}-A_{\gamma \beta a}-A_{a \gamma \beta}\right) .
\end{aligned}
$$

For the next coefficient of $\phi(x, 0)$ we find that $\nabla^{2} \phi_{0, a}$ is locally determined by $\rho_{0, a}$. However, the quantity

$$
(\phi, a \beta \gamma)_{0}-\frac{3}{5} \delta_{(\alpha \beta} \nabla^{2} \phi_{0, \gamma)}
$$

again depends on the over-all situation.

In this paper we consider the relativistic analogues of the quantities in equations (3) and (6) as adjustable parameters. This attitude is quite parochial; after all $E_{a \beta}$ is determined ultimately by the over-all matter distribution or the boundary conditions. Moreover, the number of adjustable parameters becomes uncomfortably large in our treatment. Finally, this type of purely local approach is not very appropriate in analyzing data on the distant past of our own Galaxy for cosmological significance (Hoyle 1962), or for discussing evolutionary or time-scale problems. Our approach also has some advantages. We work with measurable quantities rather than with boundary conditions that must at present be determined from philosophical or aesthetic arguments, and, of course, ignoring global properties is what makes the calculations manageable. Finally, the results are complete and quite general, and if any of our many adjustable parameters, which can all be measured in principle, can actually be observed, their values will be a very strong test for any proposed global cosmological model.

\section{ASTRONOMICAL OBSERVATIONS IN A RIEMANNIAN SPACE-TIME}

With the possible exception of very energetic cosmic rays, all of our information about the extragalactic universe comes to us as electromagnetic radiation; at present in the optical and radio regions of the spectrum, with the promise of a rapid future extension to other frequencies by observations from above the atmosphere. We must therefore begin by discussing the behavior and measurement of light in a Riemannian space-time. It might be that neutrino observations could give additional information relevant to our purposes here, but it is not obvious how. Also, we might aspire to observe the static Schwarzschild gravitational field of objects that we cannot see because they are beyond our visual horizon; such a field will in general contribute to what we have called the incident field. But it would certainly take too long to measure the incident field canonically, by measuring relative accelerations of neighboring objects, so that we must, again, look for indirect effects of the field on light beams. We will not consider here the other main body of cosmologically significant data, namely, data on the past world line of our own Galaxy (Hoyle 1962). 


\section{Geometrical Optics}

We suppose that the electromagnetic tensor for the light emitted by a distant galaxy obeys Maxwell's equations for the vacuum:

$$
F_{; b}^{a b}=0, \quad F_{[a b ; c]}=0 .
$$

The contribution of the light beams to the gravitational field is neglected. Unfortunately, exact solutions of equation (7) are not known for our general metrics. We will use a geometrical optics approximation, where the wavelength of the light is small compared with any reasonably defined radius of curvature of the universe. The following calculation is based on an idea of Trautman (1962). We assume that $F_{a b}$ can be written

$$
F_{a b}=A_{a b} e^{i S},
$$

where the derivatives of $A_{a b}$ are small, say of order $\epsilon$, and we expand $A_{a b}$ in powers of $\epsilon$ :

$$
A_{a b}=B_{a b}+\epsilon_{a b}+, \ldots, \quad B_{a b} \neq 0 .
$$

Substituting into Maxwell's equations and collecting terms of order unity and of order $\epsilon$, we find

$$
\begin{aligned}
S_{, a} B^{a b} & =0, & S_{,[a} B_{b c]} & =0, \\
B_{; b}^{a b} & =-i S_{, b} C^{a b}, & B_{[a b ; c]} & =-i S_{,[c} C_{a b]} .
\end{aligned}
$$

By writing out each component of equations (10) and (11), or by using two-component spinors, or by using a quasi-orthonormal tetrad (Jordan, Ehlers, and Sachs 1961), the reader can verify that these equations imply

and

$$
S_{, a} S^{, a}=0, \quad T_{a b}=\mu S_{, a} S_{, b}+0(\epsilon),
$$

$$
\left(\mu S^{, a}\right)_{; a}=0 \text {, }
$$

where $T_{a b}$ is the stress energy tensor of the field, and $\mu\left(x^{a}\right)$ is some scalar.

The first of equations (12) implies that the vector field $k_{a}=S_{, a}$ is tangent to a set of hypersurface-orthogonal, lightlike geodesics:

$$
k^{a} k_{a}=0, \quad k_{[a ; b]}=0=>k^{a} ; k^{b}=0 .
$$

The geodesics to which $k^{a}$ is tangent will be called "rays." The second of equations (12) shows the algebraic structure of $T_{a b}$ to lowest order. We will henceforth neglect all but this first term in $T_{a b}$. Equation (13) has a simple physical interpretation. Consider two observers who are at any two points on the same ray and who see the same (arbitrary) shift of spectral lines of the source. Suppose that each measures, at his space-time point, the infinitesimal area $d A$ and intensity $I$ of the same small bundle of rays. The rate of change of $d A$ along the ray is, in general, $k_{; a}^{a} d A$ (Jordan et al. 1961). The intensity as measured at any point on the ray by an observer with world velocity $u^{a}$ is $I=T_{a b} u^{a} u^{b}=\mu\left(k_{a} u^{a}\right)^{2}$. As we will show in the next paragraph, $k_{a} u^{a}$ for the two observers is the same, since they see no relative spectral shift. The rate of change of $I$ along the ray for all such observers is therefore just proportional to $\mu_{; a} k^{a}$, the rate of change of $\mu$, and this in turn, by equation (13), is equal to $-\mu k^{a} ; a$. It follows that the total flux of energy through $d A$ is the same for the two observers:

$$
I_{1} d A_{1}=I_{2} d A_{2} .
$$

This result does not hold if the observers see different spectral shifts. In the geometrical optics approximation, the rate of change of phase as measured by a single observer 
with world velocity $u^{a}$ is $S_{a} u^{a}=k_{a} u^{a}$. Therefore, if any two observers, at the same or different points on a single ray, measure frequencies $f_{1}$ and $f_{2}$, then

$$
f_{1} / f_{2}=\left(k_{a} u^{a}\right)_{1} /\left(k_{a} u^{a}\right)_{2} .
$$

But, as we mentioned in the preceding paragraph, the intensity measured by any observer is $\mu\left(k_{a} u^{a}\right)^{2}$. Therefore, two observers in relative motion who measure the same field at the same space-time point will measure different intensities, the ratio of intensities being the square of the ratio of the measured frequencies:

$$
I_{1} / I_{2}=\left(f_{1} / f_{2}\right)^{2} \text {. }
$$

Physically, this effect can be considered to have two causes. First, the energies of a single photon as measured by the two observers are in the ratio $f_{1} / f_{2}$; second, the unit intervals of time for the observers are in the same ratio, because of the time dilatation between the observers.

Now, instead of two instantaneously coincident observers measuring the intensities of a single source, consider the case of a single observer simultaneously measuring the intensities of two instantaneously coincident sources, of the same intrinsic luminosity (i.e., the luminosity as measured at the source in the rest frame of the source), the sources being in relative motion. The ratio of the measured intensities in this case is not the square, but the fourth power of the ratio of the measured frequencies (Jordan et al. 1961; Sachs 1961):

$$
I_{1} / I_{2}=\left(f_{1} / f_{2}\right)^{4} .
$$

Now suppose that an observer measures the bolometric intensity $I_{0}$ for light from a source of known intrinsic luminosity $L_{G}$, and that he measures the frequency $f_{0}$ for a spectral line of known intrinsic frequency $f_{G}$. We want to define a luminosity distance from source to observer, corrected for the spectral shift if $f_{0} \neq f_{G}$. But from the preceding discussion, it is clear that there are two perfectly reasonable ways to make the correction, and that these give different results. We could consider the corrected luminosity to be either (i) that which would be seen by a different observer, located at the same spacetime point but moving so that he sees no spectral shift, or (ii) that which would be seen by the same observer looking at a different source, intrinsically identical and located at the same space-time point, but with a different velocity, so chosen that the observer sees no spectral shift. We will arbitrarily make the second choice, and define the corrected luminosity distance $r$ by

$$
r^{2}=\frac{I_{G}}{I_{0}}\left(\frac{f_{0}}{f_{G}}\right)^{4}
$$

Here $I_{G}$ is the intrinsic intensity at unit distance: $I_{G}=L_{G} / 4 \pi$. This choice for $r$ has the advantage that two galaxies in collision will be assigned the same distance. For the Friedman cosmological models, the luminosity distance $r$ reduces to the "distance by apparent size" defined by McVittie (1956). This name is perhaps misleading in the present context since, as we will show shortly (cf. eq. [37]), the fact that the observed area increases as $r^{2}$ is for us a theorem rather than a definition.

2 This can also be understood physically. First, there is an intensity difference due to the relative. motion of each source and the observer. As in the case of two observers and one source, this difference comes from a difference of measured photon energies and from time dilatations, and it contributes a factor $\left(f_{1} / f_{2}\right)^{2}$ to the intensity ratio. There is also a change of intensity due to a Lorentz contraction between the sources. It is most easily seen for an observer close enough to the sources for the relevant portion of space time to look flat. The intrinsic intensity of source 1 at the location of the observer is $L / 4 \pi r_{1}{ }^{2}$, where $L$ is the intrinsic luminosity of each source and $r_{1}$ is the distance from the source to the observer as measured in the rest frame of 1 . Likewise, the intrinsic intensity of source 2 at the observer is $L / 4 \pi r_{2}{ }^{2}$. But $r_{2} / r_{1}=f_{1} / f_{2}$, so the ratio of the intrinsic intensities is $\left(f_{1} / f_{2}\right)^{2}$. The combination of these effects gives q. (18). 


\section{The Theory of Ray Bundles}

Given a distant point source of light, we will want to calculate its intensity at our Galaxy; given a distant extended source, we will want to find its apparent size and shape at our Galaxy. Both calculations involve the theory of lightlike geodesics, whose tangents $k^{a}$ obey equation (14). Since the theory has been worked out in the literature, we will just state some relevant results (Jordan et al. 1961; Sachs 1961).

Consider a point source with world velocity $u^{a}$. There is a one-parametric set of threedimensional light cones emanating from the world line of the particle. On each cone lies a two-párametric set of rays, with tangent $k^{a}$, which pass through the world line of the source. Along any ray we can define a so-called preferred affine parameter, $s$, by the equation $d x^{a} / d s=k^{a}$. For a particular light cone, $s$ is then determined up to an additive constant along the ray. If we consider the ray by itself, then $s^{\prime}=A s, k^{\prime a}=A^{-1} k^{a}$, with $A$ constant, is also an allowed transformation. Any physically measurable quantity must be invariant under these additional transformations, a fact which is useful in checking many of the following formulae for algebraic mistakes.

We now consider the particular light cone emanating from point $x_{1}{ }^{a}$ on the world line of the source. On this light cone we select an infinitesimal bundle of rays which is measured from the source to have solid angle $d \Omega$ and a circular normal cross-section. The physical picture is that someone at the source flashes a very narrow-beam flashlight on and off. Let $k^{a}$ be the tangent to the central ray $L$ of the bundle and $s$ the affine parameter on $L$, with $s=0$ at $x_{1}{ }^{a}$. Now suppose that an observer who happens to cross $L$ at some point $x_{2}{ }^{a}$ places a flat two-dimensional screen normal to the ray direction in his own rest frame. It turns out that he sees an infinitesimal, instantaneous, elliptical flash of light on the screen. The key question is: What are the size, shape, and orientation of this ellipse? It can be shown that these are all independent of the world velocity of the observer (Sachs 1961). In order to describe the ellipse, we consider the connection vectors $q^{a}$ between the central ray $L$ and the rays which define the outside of the bundle. The change of each $q^{a}$ along $L$ is described by the equation of geodesic deviation:

$$
D^{2} q^{a} / d s^{2}=-R^{a}{ }_{i b j} k^{i} k^{j} q^{b} .
$$

Here $D(\ldots) / d s=(\ldots)_{;} k^{i}$ is the absolute derivative along $L$ : we will denote it by a dot; thus, $D q^{a} / d s=\dot{q}^{a}$. The ellipse at $x_{2}{ }^{a}$ must be found by integrating equation (20) for $q^{a}$ from $x_{1}{ }^{a}$ to $x_{2}{ }^{a}$ along $L$. We need initial conditions: $q^{a}$ at $x_{1}{ }^{a}$ is zero because the cone comes to a point there. Without loss of generality, we can demand that $\dot{q}^{a}$ be orthogonal to $u^{a}$ and to $k^{a}$ at $x_{1}^{a}$ (i.e., that $\dot{q}^{a}$ be normal to $L$ in the rest frame of the source). Also, we require that the ray bundle viewed from the source has a circular cross-section and solid angle $d \Omega$. The restrictions on $q^{a}$ near $x_{1}{ }^{a}$ are thus

$$
\left(q^{a}\right)_{1}=0 ; \quad\left(\dot{q}^{a} u_{a}\right)_{1}=0 ; \quad\left(\dot{q}^{a} k_{a}\right)_{1}=0 ; \quad \pi\left(\dot{q}^{a} \dot{q}_{a}\right)_{1}=-\left(u_{a} k^{a}\right)_{1}{ }^{2} d \Omega .
$$

If we now vary the initial conditions over the values allowed by equation (21), we get from equation (20) a corresponding (one-parametric) set of vectors $\left(q^{a}\right)_{2}$ which define an ellipse at $x_{2}{ }^{a}$. The lengths $a$ and $\beta$ of the major and minor semiaxes of the ellipse are then a conditional minimum and maximum, respectively:

$$
-a^{2}=\min \left(q^{a} q_{a}\right)_{2} ; \quad-\beta^{2}=\max \left(q^{a} q_{a}\right)_{2} .
$$

The area of the ellipse can be found from equation (22), and the directions at $x_{1}{ }^{a}$ corresponding to the principal axes will of course have already been found in calculating $a$ and $\beta$. Note that, since $\left(q^{a}\right)_{2}$ is a linear function of $\left(\dot{q}^{a}\right)_{1}$, there is some two-point function $X_{b}^{a^{\prime}}\left(x_{1}, x_{2}{ }^{\prime}\right)$ for which $\left(q^{a^{\prime}}\right)_{2}=X_{b}^{a^{\prime}} \dot{q}_{1}{ }^{b}$. The maximization is a trick to avoid the need to find $X_{b}^{a^{\prime}}$ explicitly in the following calculations. 
To the accuracy needed in this paper we can explicitly do the calculation sketched above. Let $s=0$ at $x_{1}{ }^{a}$ and $s=s_{2}$ at $x_{2}{ }^{a}$. Expanding $q_{a} q^{a}$ in powers of $s$, we have

$$
\left(q_{a} q^{a}\right)_{2}=\left(q_{a} q^{a}\right)_{1}+\left[\frac{D}{d s}\left(q_{a} q^{a}\right)\right]_{1} s_{2}+\frac{1}{2}\left[D^{2}\left(q_{a} q^{a}\right) / d s^{2}\right]_{1} s_{2}^{2}+\ldots
$$

Using equation (20) and the initial conditions of equation (21), we obtain from equation (23)

$$
\left(q_{a} q^{a}\right)_{2}=s_{2}^{2}\left(\dot{q}^{a} \dot{q}^{b}\right)_{1}\left[\left(g_{a b}\right)_{1}-\frac{1}{3}\left(R_{a i b j} k^{i} k^{j}\right)_{1} s_{2}{ }^{2}-\frac{1}{6}\left(R_{a i b j ; n} k^{i} k^{i} k^{n}\right)_{1} s_{2}{ }^{3}+\ldots . .\right.
$$

Assumption (i) of the Introduction means that this series is decreasing. Since the correction term due to the curvature of space-time,

$$
t_{a b}=-\frac{1}{3}\left(R_{a i b j} k^{i} k^{j}\right)_{1} s_{2}^{2}-\frac{1}{6}\left(R_{a i b j ; n} k^{i} k^{i} k^{n}\right)_{1} s_{2}{ }^{3}+\ldots,
$$

is assumed small, we can obtain the area and shape of the ellipse at $x_{2}{ }^{a}$ by considering only terms to this order in $s_{2}$. Then, as with infinitesimal linear transformations, the contribution to the area comes only from the trace, $\left(g^{a b}\right)_{1} t_{a b}$, and the ratio of major to minor axes comes only from the trace-free part. In fact, equations (21), (22), and (24) give for the area $d A_{2}$ at $x_{2}{ }^{a}$

$$
d A_{2}=d \Omega s_{2}^{2}\left(u_{a} k^{a}\right)_{1}^{2}\left[1-\frac{1}{6}\left(R_{i j} k^{i} k^{j}\right)_{1} s_{2}^{2}-\frac{1}{12}\left(R_{i j ; n} k^{i} k^{i} k^{n}\right)_{1} s_{2}^{3}+\ldots\right] .
$$

Similarly, if $i^{a}$ is a unit vector in the direction of $\left(\dot{q}^{a}\right)_{1}$, so that $i^{a} u_{a}=i^{a} k_{a}=0$ at $x_{1}^{a}$, the ratio $e$ of major to minor axes comes out, to relevant order in $s_{2}$,

$$
e=1-\min \left(i^{a} i^{b} p_{a b}\right)+\max \left(i^{a} i^{b} p_{a b}\right)=1+2 \max \left(i^{a} i^{b} p_{a b}\right),
$$

where $p_{a b}$ contains only the conformal tensor (see eq. [64]):

$$
p_{a b}=-\frac{1}{6} s_{2}{ }^{2}\left(C_{a i b j} k^{i} k^{j}\right)_{1}-\frac{1}{12}\left(C_{a i b j ; n} k^{i} k^{i} k^{n}\right)_{1} s_{2}{ }^{3} .
$$

We shall use equations (26)-(28) to calculate luminosity distances, area distances, and the distortion effect.

We shall calculate certain observational effects which directly relate the world velocity field of the sources and the curvature to measurable quantities. In this section we shall leave the results in a form that is independent of any dynamical model or choice of coordinate system. In § III we shall specialize to general-relativistic universes and write the equations in the form that they take for an observer interpreting his data in a Newtonian, Cartesian frame of reference. In the rest of this section we consider a distant galaxy or cluster of galaxies located at a point $x_{G}{ }^{a}$ on the past light cone of the point $x^{a}=0$, which is the present space-time position of our own Galaxy. $L$ is the lightlike geodesic from the center of the galaxy or cluster to the center of an astronomer's telescope here and now ; $k^{a}$ is the tangent to $L$ and $k_{4}>0 ; s$ is an affine parameter along $L$, increasing from 0 at $x_{G}{ }^{a}$ to $s_{0}$ at the origin $x_{0}{ }^{a}=0$. A "perpendicular area" at $x_{G}{ }^{a}$ or at $x_{0}{ }^{a}$ is a two-dimensional cross-section perpendicular both to $k^{a}$ and to $u^{a}$ at the point in question. We define the following vector at $x_{0}{ }^{a}$ :

$$
e^{a}=-\left(k^{a} / u_{b} k^{b}\right)_{0}
$$

The projection of $e^{a}$ into the three-space orthogonal to $u_{0}{ }^{a}$ is a spatial unit vector in the observer's rest frame pointing in the observed direction of the source; it is given by

$$
e_{\perp}^{a}=e^{a}-u_{0}^{a}\left(e_{b} u_{0}^{b}\right)=e^{a}+u_{0}^{a} .
$$




\section{Redshift versus Luminosity Distance}

Suppose the astronomer measures frequency $f_{0}$ for a line with known intrinsic frequency $f_{G}$ emitted by the galaxy at $x_{G}{ }^{a}$. Then, from equation (16),

$$
f_{G} / f_{0}=\left(u_{a} k^{a}\right)_{G} /\left(u_{a} k^{a}\right)_{0} .
$$

Expanding in powers of $s$ gives

$$
f_{G} / f_{0}=1 /\left(u_{a} k^{a}\right)_{0}\left[u_{a} k^{a}-u_{a ; b} k^{a} k^{b} s+\frac{1}{2} u_{a ; b c} k^{a} k^{b} k^{c} s^{2}+\ldots\right]_{0} ;
$$

$s_{0}$ is not an empirically measurable quantity and must be replaced by the luminosity distance in a suitable way. We can use equation (26) with $s_{2}=s_{0}, x_{2}{ }^{a}=0$, and $d A_{2}=$ $d A_{0}$, the perpendicular area of the telescope. Let the intrinsic total luminosity of the source be $4 \pi I_{G}$ and the measured intensity be $I_{0}$. From equations (15)-(17) we get

$$
I_{0} d A_{0}\left(u_{a} k^{a}\right)_{G}^{2}=d \Omega I_{G}\left(u_{a} k^{a}\right)_{0}^{2} .
$$

From equations (19) and (26) we therefore have

$$
\begin{aligned}
r^{2} & =\left(u_{a} k^{a}\right)_{0}^{2} s_{0}^{2}\left[1-\frac{1}{6}\left(R_{i j} k^{i} k^{j}\right)_{G} s_{0}^{2}-\frac{1}{12}\left(R_{i j ; n} k^{i} k^{i} k^{n}\right)_{G} s_{0}^{3}+\ldots\right] \\
& =\left(u_{a} k^{a}\right)_{0}^{2} s_{0}^{2}\left[1-\frac{1}{6}\left(R_{i j} k^{i} k^{j}\right)_{0} s_{0}^{2}+\frac{1}{12}\left(R_{i j ; n} k^{i} k^{i} k^{n}\right)_{0} s_{0}^{3}+\ldots\right] .
\end{aligned}
$$

The reversion of this equation is

$$
s_{0}=\left(u_{a} k^{a}\right)_{0}{ }^{-1} r\left[1+\frac{1}{12}\left(R_{i j}\right)_{0} e^{i} e^{j} r^{2}+\frac{1}{24}\left(R_{i j ; k}\right)_{0} e^{i} e^{j} e^{k} r^{3}+\ldots\right] .
$$

With $s_{0}$ thus expressed in terms of $r$, equation (32) becomes

$$
f_{G} / f_{0}=1-\left(u_{a ; b}\right)_{0} e^{a} e^{b} r-\frac{1}{2}\left(u_{a ; b c}\right)_{0} e^{a} e^{b} e^{c} r^{2}+\ldots
$$

This equation contains only parameters describing the velocity field of sources and empirically measurable quantities, so that it is the desired four-dimensional formulation of the redshift versus luminosity-distance law. In the three-plus-one-dimensional notation of the next section it loses its simple form. Equation (36), but not the corresponding equation (70) of the next section, is valid in all universes in which light travels along geodesics in a Riemannian space time and obeys equation (15).

\section{Area Distance versus Luminosity Distance}

We now ask what solid angle an extended distant body subtends when viewed by the astronomer. Let its intrinsic perpendicular area be $d A_{G}$. We again use equation (26), but now our own Galaxy is located at the point called $x_{1}{ }^{a}$ and the distant object is located at the point called $x_{2}^{a}$, so the sign of $k^{a}$ must be changed. A short calculation gives

$$
d A_{G}=r^{2} d \Omega \text {. }
$$

To the accuracy we have been considering, the correction terms, which are of order $r^{4}$ and $r^{5}$, are identically zero, so that in the approximation framework, equation (37) is valid at least to terms of order $r^{6}$. R. Penrose (private communication, to be published in Hlavaty Festschrift volume) has subsequently proved, without use of power series, that equation (37) is exact.

\section{Distortion Effect versus Luminosity Distance}

It follows from § II.2 that a distant object whose intrinsic perpendicular cross-section is a circle will appear as an ellipse to us. Because $p_{a b}$ is small, the magnitude and direction of this effect can be read off directly from equation (27), using the same conventions as in 
$\S$ II.4. We again designate a unit spatial vector in the astronomer's laboratory and tangent to the astronomer's unit sphere by $i^{a}$. Then, from equations (28) and (35), the ratio, $e$, of major to minor axis of the observed ellipse is

$$
e=1+\frac{1}{3} \max \left(i^{a} i^{b} e^{i} e^{i} C_{a i b j}\right)_{0} r^{2}+\ldots
$$

where the maximum is taken by varying the end point of $i^{a}$ over the spatial unit circle orthogonal to the source direction $e_{\perp}{ }^{a}$ in the astronomer's rest frame; the direction of the major axis is of course that $i^{\alpha}$ for which the maximum occurs.

\section{Number Counts versus Luminosity Distance}

Suppose we count the number $d N$ of galaxies in a solid angle $d \Omega$ (measured by the astronomer) which have luminosity distances between $r$ and $r+d r$. To $d r$ corresponds some parameter interval $d s$ and also some infinitesimal length $d x$ orthogonal to the intrinsic perpendicular cross-section $d A_{G}$ at $x_{G}{ }^{a}$. Then for $d N$ we find $d N=\left(\rho^{\prime} d A d x\right)_{G}$, where $\rho^{\prime}$ is the intrinsic number of galaxies per unit volume. On the other hand, we already know $d A_{G}=d \Omega r^{2}$; moreover, a short calculation gives $d x=d s\left(u_{a} k^{a}\right)_{G}$. Using these equations and the differential of equation (35), we get

$$
d N=\left(f_{G} / f_{0}\right) r^{2} d \Omega d r\left[\rho_{0}^{\prime}+\left(\rho_{, a}^{\prime}\right)_{0} e^{a} r+\frac{1}{2} e^{a} e^{b} r^{2}\left(\rho_{; a b}^{\prime}+\frac{1}{2} R_{a b} \rho^{\prime}\right)_{0}+\ldots\right] .
$$

We could also expand the redshift correction $\left(f_{G} / f_{0}\right)$ in powers of $r$, but it seems more useful to consider it as an empirical quantity as far as equation (39) is concerned.

\section{Apparent Proper Motions versus Luminosity Distance}

If an astronomer at $x^{a}=0$ measures the apparent direction, with respect to a local set of non-rotating axes, of a distant object at $x_{G}{ }^{a}$, it may happen that this direction changes in the course of time. Such an apparent proper motion may arise, loosely speaking, either from a "real" motion of the source or from a curvature of space time between the source and the observer. To calculate this effect, we must examine the lightlike geodesic that starts at the point $x_{1}^{a}=x_{G}{ }^{a}+\epsilon u_{G}{ }^{a}$, where $\epsilon$ is infinitesimal, and intersects the observer's world line at the point $x_{2}{ }^{a}=u^{a} \Delta t$, where $\Delta t$ is an infinitesimal increase in the observer's proper time. Note that $\Delta t / \epsilon=f_{G} / f_{0}$, and also that, by equation (16), $f_{G} / f_{0}=\left(u_{a} k^{a}\right)_{G} /$ $\left(u_{a} k^{a}\right)_{0}$.

Suppose that the tangent to this geodesic is $m^{a}=d x^{a} / d s$, where $s=0$ at $x_{1}{ }^{a}$ and $s=s_{2}$ at $x_{2}{ }^{a}$. In analogy with $e^{a}$, we define the vector

$$
f^{a}=-\left(m^{a} / u_{b} m^{b}\right)_{2} \text {. }
$$

This is a vector at $x_{2}{ }^{a}$ whose projection, into the three-space orthogonal to $u_{2}{ }^{a}$, is a unit spatial vector in the observer's rest frame pointing in the observed direction of the source at time $t_{0}+\Delta t$.

We suppose henceforth that the observer moves along a geodesic, as is the case; for example, in a relativistic dust model. Consider an orthonormal tetrad $\lambda_{(a)}{ }^{i}$ on the observer's world line, where $\lambda_{(4)}{ }^{i}=u^{i}$ and the triad $\lambda_{(a)}{ }^{i}(a=1,2,3)$ is parallel-transferred along the observer's world line, and therefore forms a set of non-rotating Cartesian axes for the observer. (Note that the numbers and letters in parentheses are labels for the vectors, not tensor indices.) Such a vector might be the axis direction of our own Galaxy, treated as a gyroscope, corrected for torques exerted by local irregularities. We shall return to the question of establishing a non-rotating frame in $\S$ III, and will show there that the somewhat unrealistic appeal to a local gyroscope can be avoided in practice. (See the discussion following eq. [86].) The direction cosines of the source at times $t_{0}$ and $t_{0}+\Delta t$ are

$$
e_{(a)}=-e^{i}\left(\lambda_{(a)}\right)_{0} \quad \text { and } \quad f(a)=-f^{i}\left(\dot{\lambda}_{(a)_{i}}\right)_{2},
$$


respectively. In general, $e_{(a)} \neq f_{(a)}$, and their difference measures the source's apparent proper motion, defined as

$$
\frac{d e(a)}{d t}=\lim \frac{f(a)-e(a)}{\Delta t} .
$$

Expanding $\left(\lambda^{i}(a)\right)_{2}$ about $x^{a}=0$, and noting that $f^{i}=e^{i}+0(\Delta t)$, this becomes

$$
-\frac{d e(a)}{d t}=\lim \frac{\left(f^{i}-e^{i}\right)\left(\lambda(a)_{i}\right)_{0}}{\Delta t}+\left(\Gamma_{j k}^{i} e^{j} u^{k} \lambda_{(a)_{i}}\right)_{0} .
$$

It then remains only to expand $f^{i}$ about $x^{a}=0$, which we will do by expanding $m^{a}$ and $u_{a}$ separately, and then combining them according to equation (40).

Since the observer moves on a geodesic,

$$
\left(u_{b}\right)_{2}=\left(u_{b}\right)_{0}+\left(\Gamma_{b c}^{d} u_{d} u^{c}\right)_{0} \Delta t+O\left[(\Delta t)^{2}\right] .
$$

To expand $m_{2}{ }^{a}$, we first write it in terms of $\Delta x^{a}=x_{2}{ }^{a}-x_{1}{ }^{a}$. Thus, since $m^{a}$ is the geodesic joining $x_{1}^{a}$ and $x_{2}^{a}$,

$$
x_{1}^{a}=x_{2}^{a}-s_{2} m_{2}^{a}-\frac{1}{2} s_{2}{ }^{2}\left(\Gamma_{b c}{ }^{a} m^{b} m^{c}\right)_{2}+\ldots
$$

The reversion of this series is

$$
s_{2} m_{2}^{a}=\Delta x^{a}-\frac{1}{2}\left(\Gamma_{b c}{ }^{a}\right)_{2} \Delta x^{b} \Delta x^{c}+O\left[(\Delta x)^{3}\right] .
$$

Note further that

and that

$$
x_{2}^{a}=u_{0}^{a} \Delta t+O\left[(\Delta t)^{2}\right],
$$

$$
x_{1}^{a}=x_{G}^{a}+\epsilon \mathcal{U}_{G}^{a}+O\left(e^{2}\right)=x_{G}^{a}+\left(f_{0} / f_{G}\right) u_{G}^{a} \Delta t+O\left[(\Delta t)^{2}\right] .
$$

The rest of the calculation is straightforward, though somewhat tedious. Equation (36) gives $f_{G} / f_{0}$ as a power series in $r$, and if we also expand $x_{G}{ }^{a}$ and $u_{G}{ }^{a}$ in powers of $r$, then equations (46)-(48) give $m_{2}{ }^{a}$ as a power series in $r$ and $\Delta t$. Substituting this and equation (44) into equation (40) gives $f^{a}$ as a series in $r$ and $\Delta t$. Finally, equation (43) then gives the apparent proper motion of the source as a series in $r$. The result of this calculation is

$\frac{d e(a)}{d t}=-h{\left({ }_{\alpha \beta}\right)}_{t}\left[\left(u_{; b}^{i}\right)_{0} e^{b}+r\left(\frac{1}{2} u_{; j k}^{i}-\frac{1}{2} R_{\cdot j k b}^{i} u^{b}+u_{;}^{i} e^{b} u_{j ; k}\right)_{0} e^{j} e^{k}+O\left(r^{2}\right)\right]\left(\lambda(\beta)_{i}\right)_{0}$.

Here $h_{(a \beta)}=\delta_{a \beta}-e_{(\alpha)} e_{(\beta)}$ is a projection operator into the plane orthogonal to $e$ : the right-hand side of equation (49) is summed over $\beta$.

Equations (36)-(39) and (49) are the fundamental observational laws of this paper. In the next section we shall specialize them to general-relativistic universes and write them in a three-plus-one dimensional notation.

\section{OBSERVATIONS IN DUST-FILLED GENERAL-RELATIVISTIC UNIVERSES}

In $\S$ II we have derived general expressions in power series for five kinds of astronomical observations in a Riemannian space time. The coefficients of the series are the density and velocity fields of matter, the curvature of space time, and their derivatives, all evaluated here and now. These quantities are, of course, determined by the over-all structure of the universe, but they are here treated as parameters whose values must be found, if possible, from observations. For any particular gravitational theory, the coefficients will not be independent. As an example we shall consider Einstein's gravitational theory for a dust-filled universe, without making any assumptions about the 
symmetry of the cosmos. We suppose therefore that the Einstein tensor, $G^{a b}=R^{a b}-$ $\frac{1}{2} g^{a b} R=R^{a i b} i-\frac{1}{2} R^{i j} \ddot{i j}^{a b}$, has the algebraic form

$$
G^{a b}=\rho u^{a} u^{b}+\Lambda g^{a b} ; \quad u^{a} u_{a}=1 .
$$

Here $\rho$ is the proper density of energy in natural units, $u^{a}\left(x^{i}\right)$ is the average world velocity of the galaxies near the point $x^{i}$, and $\Lambda$ is the cosmological constant. A continuous distribution of $\rho$ and $u^{a}$ is assumed. The cosmological constant is retained merely for the sake of generality; it plays little role in the following.

We shall also use a system of Riemannian normal coordinates (Birkhoff 1923), referred to our present space-time position, with the time axis along our local time axis. That is, if $\left(u^{a}\right)_{0}$ is the average velocity of galaxies in our immediate vicinity now, then

$$
\left(u^{a}\right)_{0}=\delta_{4}^{a} \text {. }
$$

Riemannian normal coordinates have two defining properties: (i)

$$
\left(g_{a b}\right)_{0}=\eta_{a b}
$$

where $\eta_{a b}=\eta^{a b}$ is the diagonal Lorentz metric with components $(-1,-1,-1,+1)$; (ii) every geodesic through the origin has, at each of its points, the equation $d^{2} x^{a} / d s^{2}=0$. Detailed discussions of Riemannian coordinates will be found in the literature (Thomas 1934). Here we mention only two properties of interest in this paper. First, under very reasonable smoothness assumptions, Riemannian normal coordinates exist, but usually only in a finite region around the origin; our fundamental assumption that all quantities can be expanded in power series therefore must include the assumption that we are well within this region. Second, under the condition given by equation (51), the Riemannian normal coordinates are unique up to the Euclidean transformations

$$
\bar{x}^{\beta}=O_{a}^{\beta} x^{\alpha}, \quad \bar{t}=t, \quad(a, \beta=1,2,3),
$$

where $O_{a}{ }^{\beta}$ is a constant orthogonal matrix.

These special coordinates have been chosen primarily for computational convenience. They also have the advantage that the theoretical observational effects which we will write in terms of them can be compared directly with observational results as usually reported. The coordinates used by an observational astronomer are $t$, the time of observation of an event; $e_{(a)}$ (eq. [41]), the direction cosines of the event referred to a non-rotating orthonormal triad $\lambda_{(a)}{ }^{i}$ in the observer's rest frame; and $r$, the luminosity distance of the source. But the tangents to the parametric lines of the space coordinates $x^{a}$ of our Riemannian normal coordinates form an orthonormal triad in the observer's rest frame, and this triad can always be made to coincide with the observer's reference frame $\lambda_{(a)}{ }^{i}$ by a transformation defined by equation (53). If this is done, it is easy to show that $e_{(a)}=$ $e^{a}$; i.e., that the contravariant component $e^{a}$ is numerically the same as the corresponding direction cosine as measured by an astronomer. Therefore, when we come to write equations such as (71), $e^{a}$ can be interpreted directly as a measured angle.

The calculations to follow could easily be done under assumptions other than equation (50); i.e., for other gravitational theories than Einstein's. The results, including the distortion effect discussed in $\S$ II.5, would be similar for any cosmology which assumes that light travels along geodesics in a Riemannian space-time. However, as we shall see. there are many adjustable parameters in our final results even with this assumption,

\section{The Independent Parameters}

We shall now systematically determine which of the parameters $\rho, u^{a}, R_{a b c d}$, and their derivatives at $x^{a}=0$ can be chosen independently. The reader who starts to get lost in the details of the calculations may find it helpful to refer to Table 1 (see below). 
From the twice-contracted Bianchi identities, $G^{a b}{ }_{; b}=0$, we have

$$
\left(\rho u^{a}\right)_{; a}=0, \quad u_{; b}^{a} u^{b}=0 .
$$

The first of these is the conservation equation; the second says that matter moves along geodesics. Also, $u^{a} u_{a}=1$ implies

$$
u^{a} ; b u_{a}=0 \text {. }
$$

The first free parameter is $\rho_{0}$. Consider next in Riemannian normal coordinates $\left(u_{a ; b}\right)_{0}=\left(u_{a, b}\right)_{0}$. From equations $(51),(52),(54)$, and (55) we obtain $\left(u_{4 ; a}\right)_{0}=0,\left(u_{a ; 4}\right)_{0}=$ 0 . Consequently, only the $3 \times 3=9$ constants $u_{a \beta}$ defined by

$$
u_{\alpha \beta}=\left(-u_{a ; \beta}\right)_{0}
$$

are at our disposal. These have a direct analogue in Newtonian theory. If $v_{a}$ is the velocity of matter in Newtonian theory, then $\left(v_{a, \beta}\right)_{0}$ is fully analogous to $\left(-u_{a ; \beta}\right)_{0}$. Also, if the astronomer interprets $X^{a}=e_{(a)} r$ as a Cartesian vector and defines the corresponding "velocity" $V^{a}=d X^{a} / d t$, then $\partial V^{a} / \partial X^{\beta}=u_{a \beta}$. Therefore we split $u_{a \beta}$, in the familiar way, into a rotation $\omega_{a \beta}$, a shear $\sigma_{a \beta}$, and an expansion $\theta$ :

Here

$$
u_{\alpha \beta}=\omega_{a \beta}+\sigma_{a \beta}+\frac{1}{3} \delta_{\alpha \beta} \theta \text {. }
$$

$$
\omega_{\alpha \beta}=u_{[\alpha \beta]}, \quad \theta=u_{a a}, \quad \sigma_{a \beta}=u_{(a \beta)}-\frac{1}{3} \delta_{a \beta} \theta .
$$

Note that such quantities as $\delta_{a \beta}$ and $u_{a \alpha}$ are well defined because of the fact that the only allowed coordinate transformations are the rigid Euclidean rotations of equation (53).

[The following is intended purely as an aside to those readers who are acquainted with representation theory. Note that what we have really done is to split $\left(u_{a ; b}\right)_{0}$ into six quantities $\left[\left(u_{4 ; 4}\right)_{0},\left(u_{4 ; a}\right)_{0},\left(u_{a ; 4}\right)_{0}, \omega_{a \beta}, \sigma_{a \beta}\right.$, and $\left.\theta\right]$, each of which forms an irreducible representation of the rotation group, equation (53). The first three vanish and the last three are at our disposal. Very similar considerations are the basis for all the remaining calculations in this section. In some cases we will find it convenient to carry the splitting only part way by combining several irreducible representations in one symbol. But any doubt concerning the arbitrariness of a given coefficient can always be settled systematically by using two-component spinors, or by using Young's diagrams and subtracting out suitable traces (Murnaghan 1938; Penrose 1960).]

Proceeding to $(\rho, a)_{0}$ we find that the three quantities $(\rho, a)_{0}$ are arbitrary while $(\rho, 4)_{0}$ is given by equation $(54):\left(\rho_{, 4}\right)_{0}=-\rho_{0} \theta$. Next consider $\left(u_{a ; b c}\right)_{0}$. There are $3 \times(3 \times 2 / 2)$ $=9$ free quantities,

$$
u_{\mu \beta \delta}=-\left(u_{\mu ;(\beta \delta)}\right)_{0} .
$$

The other components of $\left(u_{a ; b c}\right)_{0}$ are determined from our previous relations:

$$
\begin{gathered}
2\left(u_{\mu ;[\beta \delta]}\right)_{0}=\left(R_{4 \mu \beta \delta}\right)_{0}, \quad\left(u_{4 ; \beta \delta}\right)_{0}=u_{\tau \beta} u_{\tau \delta}, \quad\left(u_{\beta ; 4 \delta}\right)_{0}=u_{\beta \tau} u_{\tau \delta}, \\
\left(u_{\beta ; \delta 4}\right)_{0}=u_{\beta \tau} u_{\tau \delta}+\left(R_{4 \beta \delta 4}\right)_{0}, \quad\left(u_{4 ; 44}\right)_{0}=\left(u_{\mu ; 44}\right)_{0}=\left(u_{4 ; \mu 4}\right)_{0}=\left(u_{4 ; 4 \mu}\right)_{0}=0 .
\end{gathered}
$$

The Riemann tensor terms in equation (60) will be analyzed presently. For the moment we consider them as given.

The constants $u_{\mu \beta \delta}$ of equation (59) "essentially" represent differential rotations, shears, and expansions; they correspond "essentially" to $v_{\mu, \beta \delta}$ of Newtonian theory. But there is an important ambiguity in this correspondence. We could just as well draw the analogy

$$
v_{\mu, \beta \delta} \leftrightarrow\left(-u_{\mu ;(\beta \delta)}\right)_{0}+a\left(R_{4(\beta \delta)_{\mu}}\right)_{0},
$$


where $a$ is any pure number (of order unity), since $R_{4(\beta \delta)_{\mu}}$ has the same dimensions and symmetries as $v_{\mu, \beta \delta}$ and vanishes in the correspondence limit $R_{a b c d} \rightarrow 0$. In the authors' opinion it would be futile to call any particular one of the quantities in equation (61) the unique analogue of $v_{\mu, \beta \delta}$. For example, $u_{\mu, \beta \delta}$ (which is well defined because our coordinate transformations are so restricted) differs from $u_{\mu ;(\beta \delta)}$ as in equation (61), but in all other respects is on the same footing. This means that any attempt to divide observational results unambiguously into curvature effects and kinematic or dynamic effects will fail for sufficiently complicated effects. Once we accept such ambiguities as inevitable they do no real harm.

Next, among the second derivatives of $\rho$, the six quantities

$$
\rho_{a \beta}=\left(\rho_{; \alpha \beta}\right)_{0}
$$

are arbitrary. The remaining second derivatives are given by

$$
\begin{gathered}
\left(\rho_{; 4 \beta}\right)_{0}=\left(\rho_{; \beta 4}\right)_{0}=-\left(\rho_{, \mu}\right)_{0} u_{\mu \beta}-\left(\rho_{, \beta}\right)_{0} \theta-\rho_{0} u_{\mu \mu \beta}, \\
\left(\rho_{; 44}\right)_{0}=\rho_{0}\left[\theta^{2}+u_{\beta \delta} u_{\delta \beta}+\frac{1}{2} \rho_{0}-\Lambda\right] .
\end{gathered}
$$

Note that if $\omega_{\mu \beta}=0$ and $\Lambda=0$, then $\left(\rho_{; 44}\right)_{0}>0$. We have enough terms now in the expansions of $\rho$ and $u_{a}$ and can proceed to the curvature tensor.

In the lowest order, $\left(R_{a b c d}\right)_{0}$ splits into the Ricci tensor $\left(R_{a b}\right)_{0}$ and the conformal (or Weyl) tensor $\left(C_{a b c d}\right)_{0} ; C_{a b c d}$ is defined as

$$
C_{* c d}^{a b}=R_{* c d}^{a b}-2 \delta_{[c}^{[a} R_{d]}^{b]}+\frac{1}{3} \delta{ }_{c}^{[a} \delta_{d}^{b]} R .
$$

The ten components of the Ricci tensor are determined by the field equations (50):

$$
\left(R_{a b}\right)_{0}=\left(G_{a b}\right)_{0}-\frac{1}{2} \eta_{a b} G_{0}=\rho_{0} \delta_{a}^{4} \delta_{b}^{4}-\frac{1}{2} \eta_{a b}\left[2 \Lambda+\rho_{0}\right] .
$$

$\left(C_{a b c d}\right)_{0}$ is the incident field; its ten components are at our disposal. As is well known (Pirani 1962a,b), $\left(C_{a b c d}\right)_{0}$ can be further split into two quantities $E_{\mu \beta}$ and $H_{\mu \beta}$ :

$$
E_{\mu \beta}=\left(C_{4 \mu 4 \beta}\right)_{0} ; \quad H_{\mu \beta}=\frac{1}{2}\left(C_{4 \mu \rho \sigma}\right)_{0} \epsilon_{\rho \sigma \beta},
$$

where $\epsilon_{\rho \sigma \beta}$ is the three-dimensional Levi-Civita object. $E_{\mu \beta}$ is manifestly trace-free and symmetric and, as shown in the literature (Pirani $1962 a, b), H_{\mu \beta}$ has the same properties;

$$
E_{\mu \beta}=E_{\beta \mu}, \quad E_{\mu \mu}=0 ; \quad H_{\mu \beta}=H_{\beta \mu}, \quad H_{\mu \mu}=0 .
$$

$E_{\mu \beta}$ is usually called the incident "electric-type" field. In the usual slow-motion, weakfield approximation, $E_{\mu \beta}$ goes into the Newtonian quantity $\left(\phi_{, \mu \beta}\right)_{0}-\frac{1}{3} \delta_{\mu \beta} \nabla^{2} \phi_{0}$, which we discussed in $\$ \mathrm{I}$. $H_{\mu \beta}$ has no Newtonian analogue; it is usually called the incident "magnetic-type" field. One reason for this name is that, in linearized gravitational theory, contributions to $H_{\mu \beta}$ come primarily from rotations of the sources of the field (Sachs and Bergmann 1958).

There are, intuitively speaking, several possible sources of the incident field, such as asymmetries in the large-scale matter or velocity distribution of galaxies, or in the boundary conditions for the universe. Also, gravitational waves with periods of the order of $10^{9}$ years would contribute to the incident field; waves whose periods are a mere $10^{6}$ years or so would be considered in our treatment as local irregularities, and would be averaged out. Since the methods of this paper cannot be used to analyze global questions, the incident field is, from our point of view, a purely empirical quantity. In § III.2, we shall crudely estimate the maximum size of $\left(C_{a b c d}\right)_{0}$ as about $10^{-16}$ (light-year) ${ }^{-2}$ on the basis of existing data. 
We might point out that the list of independent parameters in Table 1 (see below) is complete to the order considered. In particular, it can be shown that the first derivatives of the metric tensor vanish at $x^{a}=0$, and all of the second derivatives are calculable from the Riemann tensor.

\section{Results and Discussion}

At the cost of some repetition, this section is intended to be understandable to a reader who has no detailed knowledge of relativity theory or has not read $\$ \S$ II and III.1. An understanding of Cartesian (Euclidean) tensors is assumed, but the reader who feels uneasy with tensors can get a qualitative idea of the results by thinking of quantities with more than one index as coefficients of certain spherical harmonics, as indicated in equation (72). All tensors in this section are to be interpreted as Cartesian tensors in the Euclidean frame of the astronomer's observatory. All indices run from 1 to 3 ; for consistency with previous sections, certain indices will be placed as superscripts rather than subscripts, but this placement has no relevance as far as this section alone is concerned.

In our units, $c=1$, the Newtonian gravitational constant is $1 / 8 \pi$, and the Einstein gravitational constant is consequently 1 . All quantities then have the dimensions of time to some power. Thus a length of $10^{9}$ years means a length of $10^{9}$ light-years.

The astronomer points his telescope along the unit vector $e$, with Cartesian components $e^{a}$, toward some distant source, such as a galaxy or cluster of galaxies. The "intrinsic" frequency of a line at the source (i.e., the frequency measured by a hypothetical observer at the source, moving with the source at the time the source emits the light) is $f_{G}$. The total intrinsic luminosity of the source is $L_{G}$. For simplicity, the source is assumed intrinsically spherical with intrinsic cross-sectional area $d A_{G}$. It is assumed that $f_{G}, L_{G}$, and $d A_{G}$ are somehow known. We define $I_{G}=L_{G} / 4 \pi$.

The astronomer measures various quantities. We shall suppose that all of them have been corrected in the usual way for the motion of the Earth relative to the center of mass of the local group of galaxies; no correction of this type is needed to analyze the distortion effect because two relatively moving observers in the same place will measure the same shape (though not the same size) for the distant object.

The astronomer measures a frequency $f_{0}$. He measures an intensity and then corrects it for absorption to get the bolometric intensity $I_{0}$. If the intensity is measured only over some limited frequency range, then the fact that a redshift has occurred must be taken into account in extrapolating to bolometric intensity; but no additional redshift corrections are to be incorporated into $I_{0}$. He measures by Euclidean methods the solid angle $d \Omega_{0}$ subtended by the distant object. He sees the intrinsically spherical distant object as an ellipse and measures the orientation and shape of the ellipse on his plates; if the distant object is irregular, he measures the orientation of its longest dimension and the ratio of longest to shortest dimension for use in a statistical treatment. He counts the number $d N$ of galaxies or groups in a given solid angle and a given intensity range. He measures the rate of change $d \boldsymbol{e} / d t$ of the source's direction relative to a non-rotating, mutually orthogonal set of directions (such as the axial direction of our own Galaxy after correction for torques exerted by members of our own galactic group; cf. eq. [86] and the discussion following it). He defines the "corrected luminosity distance" $r$ as

$$
r=\left(I_{G} / I_{0}\right)^{1 / 2}\left(f_{0} / f_{G}\right)^{2} .
$$

As discussed in $\S I I .1$, it is convenient to incorporate into $r$ the particular redshift correction given by equation (68).

The equations in this section take their simplest form if we plot all effects as functions of $r$. But since the redshift is by far the most accurately observed quantity, we shall also write some of the equations in more conventional form, with $z=\left(f_{G} / f_{0}\right)-1$ as independent variable.

We assume that the gravitational field obeys Einstein's gravitational equations for 
dust. All small-scale variations in the density or velocity of the dust are averaged out, but inhomogeneities whose distance scales are of the order of $10^{9}$ years or larger are allowed. We work in a frame for which the three-dimensional velocity of the dust here and now is zero. The observable effects are determined, to the order of accuracy considered, by certain parameters; these parameters are listed in Table 1. Except for the algebraic restrictions given in the table, the parameters are free and independent in a general relativistic universe without pressure, and we consider them as quantities whose values must be found from observations. The number of free parameters can of course be reduced if additional a priori assumptions are made about the character of the metric. Such assumptions greatly simplify the mathematics, but current observations do not unambiguously suggest any particular simple model, including the Friedman model (cf.

TABLE 1

The Adjustable Parameters

\begin{tabular}{|c|c|c|c|}
\hline Symbol & Name & Newtonian Analogue & $\begin{array}{l}\text { Relativistic } \\
\text { Definition }\end{array}$ \\
\hline 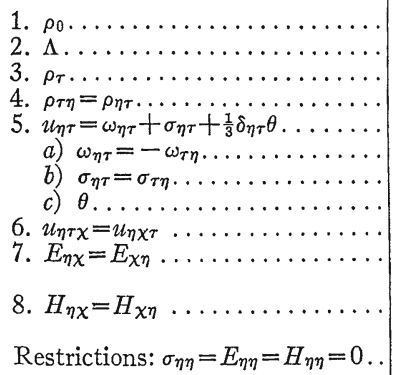 & 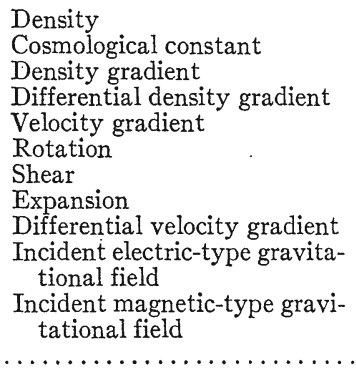 & $\begin{array}{l}\left.\rho_{0}=\rho\right]_{x=t=0} \\
\text { None } \\
\left.(\rho, \tau)_{0}=\partial \rho / \partial x^{\tau}\right]_{X=t=0} \\
(\rho, \eta \tau)_{0} \\
\left(v_{\eta, \tau}\right)_{0} * \\
(v[\eta, \tau])_{0} \\
(v(\eta, \tau))_{0}-\frac{1}{3} \delta_{\eta \tau} \nabla \cdot v_{0} \\
\nabla \cdot v_{0} \\
\left(v_{\eta, \chi \tau}\right)_{0} \\
(\phi, \eta \chi)_{0}-\frac{1}{3} \delta_{\eta \chi} \nabla^{2} \phi_{0} \\
\text { None }\end{array}$ & $\begin{array}{l}\text { Eq. }(50) \\
\text { Eq. }(50) \\
(\rho, \tau) \\
\text { Eq. }(62) \\
\text { Eq. }(56) \\
\text { Eq. (58) } \\
\text { Eq. (58) } \\
\text { Eq. (58) } \\
\text { Eq. (59) } \\
\text { Eq. (66) } \\
\text { Eq. (66) }\end{array}$ \\
\hline
\end{tabular}

$* v$ is the velocity of the dust in Newtonian approximation and $v_{0}=0$.

eq. [88]). In the Friedman case, the universe is assumed to be homogeneous and isotropic, and the following equations are found to hold:

$$
\rho_{\mu}=\sigma_{\mu \nu}=\omega_{\mu \nu}=u_{\mu \nu \gamma}=E_{\mu \nu}=H_{\mu \nu}=0, \quad \rho_{\mu \nu}=\frac{1}{3} \rho_{0} \theta^{2} \delta_{a \beta},
$$

so there are only three free parameters. If we make the transformation from Riemannian normal coordinates to the usual Robertson-Walker coordinates for the Friedman universe, we can establish the following identifications, which will be useful in examining our later results in the Friedman limit:

$$
\theta=3 h_{1}, \quad \rho_{0}=2\left(h_{1}{ }^{2}-h_{2}+k\right), \quad \Lambda=h_{1}{ }^{2}+2 h_{2}+k .
$$

The quantities $h_{1}, h_{2}$, and $k$ are defined for the Robertson-Walker metric in McVittie (1956): $h_{1}$ and $h_{2}$ are the Hubble parameter and the acceleration parameter, respectively.

We shall now discuss the dependence of the redshift on $r$, on the direction of observation $e$, and on the parameters in Table 1. The $r$-dependence of $z$ has the form (eq. [36])

$$
z=W r+\frac{1}{2} X r^{2}+\ldots
$$

The angular dependence of $W$ is given by (cf. Ehlers 1961)

$$
W=\frac{1}{3} \theta+\sigma_{\mu \gamma} e^{\mu} e^{\gamma} \text {. }
$$


The $\theta$-term in equation (71) is spherically symmetric ( $\theta$ is the expansion of the universe, not an angle); the five linearly independent components of $\sigma_{\mu \gamma}$ correspond essentially to the five linearly independent coefficients $\sigma_{m}$ of a sum

$$
\sum_{m=-2}^{+2} \sigma_{m} Y_{2 m}(\Theta, \phi) \text {. }
$$

Here $\theta$ and $\phi$ are angles on the celestial sphere and $Y_{n m}$ are surface harmonics. Similarly, $X$ contains surface harmonics of order $0,1,2$, and 3:

$$
X=V+V_{\mu} e^{\mu}+V_{\mu \gamma} e^{\mu} e^{\gamma}+V_{\mu \beta \gamma} e^{\mu} e^{\gamma} e^{\beta} .
$$

The tensors in this equation are defined in terms of the parameters by

$$
\begin{gathered}
V=\frac{1}{6} \rho_{0}+\frac{1}{3} \theta^{2}+\sigma_{\mu \gamma} \sigma_{\mu \gamma}-\frac{1}{3}\left(\Lambda+\omega_{\mu \gamma} \omega_{\mu \gamma}\right), \quad V_{\mu}=\frac{1}{5}\left(2 u_{\gamma \gamma \mu}+u_{\mu \gamma \gamma}\right), \\
\left.-V_{\mu \gamma}=E_{\mu \gamma}+\omega_{\mu \lambda \omega_{\gamma \lambda}+2 \omega_{\mu \lambda} \sigma_{\gamma \lambda}-3 \sigma_{\mu \lambda} \sigma_{\gamma \lambda}-2 \sigma_{\mu \lambda} \theta-\delta_{\mu \gamma}\left(\frac{1}{3} \omega_{\lambda \beta} \omega_{\lambda \beta}-\sigma_{\lambda \beta} \sigma \lambda \beta\right.}\right), \\
V_{\mu \gamma \lambda}=u_{\mu \gamma \lambda}-\frac{1}{5}\left(u_{\mu \beta \beta}+2 u_{\beta \beta \mu}\right) \delta_{\gamma \lambda} .
\end{gathered}
$$

Equations (70), (71), (73), and (74) give the desired expression for $z$.

To compare with available observations, it is convenient to consider the Friedman models, for which equation (70) reduces to

$$
z=h_{1} r+\frac{1}{2}\left(3 h_{1}^{2}-h_{2}\right) r^{2}+\ldots
$$

This is equation (9.203) in McVittie (1956). In comparing these and other equations in the Friedman limit, note that

$$
r=\frac{D}{2}\left[1-2 h_{1} \frac{D}{c}+\left(4 h_{1}^{2}+h_{2}\right) \frac{D^{2}}{c^{2}}+\ldots\right],
$$

where $D$ is defined in McVittie's equation (8.517). Although $h_{1}$ and $h_{2}$ are spherically symmetric while $W$ and $X$ in equation (70) have the angular dependence indicated in equations (71) and (73), we can use the empirical values of $h_{1}$ and $h_{2}$ to get some very rough estimates or upper bounds for the various parameters that appear in equations (71) and (74).

As far as the linear term $W$ is concerned, we first remark that $W$ has the structure of equation (71) for most current cosmological theories, not just for general relativistic dust models. If any spherical harmonic of order one, such as an asymmetry between north and south galactic poles, were observed in the linear portion of the redshift-curve, this would indicate a non-zero acceleration $\left(u_{a ; 4}\right)_{0} \neq 0$. In general relativistic models, such accelerations arise only from pressure gradients. Of course, a north-south asymmetry which is independent of $r$ would simply indicate motion relative to the center of mass of the local group of galaxies. Since the observed linear redshift is, at least very roughly, spherically symmetric, we take the current observational value (Sandage 1962 ), $h_{1} \approx$ $10^{-10}$ year $^{-1} \approx 100 \mathrm{~km} / \mathrm{sec} / \mathrm{Mpc}$, to indicate $\theta \approx 3 \times 10^{-10}$ year $^{-1}$. The authors have not been able to find observational estimates of the second spherical harmonic component in the linear redshift curve. However, a brief examination of the published cluster data (Humason, Mayall, and Sandage 1956) makes it appear likely that

$$
\sigma_{\mu \gamma} \leq 5 \times 10^{-11} \text { year }^{-1},
$$

since a value of $\sigma_{\mu \gamma}$ larger than this would by itself produce a larger dispersion in the redshift-magnitude relation than is observed. In the absence of a more systematic esti- 
mate we shall take equation (77) as the upper bound for $\sigma_{\mu \gamma}$; this number is to be considered with even more caution than our previous estimates.

Turning now to the quadratic term in equatio.s (70) and (75), there seems little hope of guessing which spherical harmonics in $X$ have actually been measured. To get numerical estimates we arbitrarily assume that no large accidental numerical cancellations have occurred in $X$ for the particular directions in which $h_{2}$ has been measured. For the numerical value of $h_{2}$ we choose (Sandage 1961)

$$
0 \geq h_{2} \geq-2 h_{1}^{2} \approx-2 \times 10^{-20} \text { year }^{-2} .
$$

This limit is of course much less accurate than the estimate of $h_{1}$. We then get the following upper bounds:

$$
\begin{aligned}
\Lambda, E_{\mu \gamma}, u_{(\mu \gamma \lambda)} & \lesssim 2 \times 10^{-20} \text { year }^{-2}, \quad \omega_{\mu \gamma} \lesssim 10^{-10} \text { year }^{-1}, \\
\rho_{0} & \lesssim 10^{-19} \text { year }^{-2} \approx 10^{-28} \mathrm{gm} / \mathrm{cm}^{3} .
\end{aligned}
$$

It is important to the following discussion that $H_{\mu \gamma}$ does not appear in the redshift formula, so that it is not bounded by the redshift measurements. The same is true of the part of $u_{\mu \gamma \lambda}$ that is antisymmetric in the first two indices.

On occasion, much has been made of the fact that the acceleration parameter appears to be negative and of order unity. If we are willing to consider inhomogeneities or anisotropies, then the present measurements are far from decisive. It may simply be that $E_{\mu \gamma}$ or $u_{(\mu \gamma \lambda)}$ makes a contribution of the required magnitude for the particular directions in which the acceleration parameter has been measured; since measurements in the galactic plane are difficult and few, the angular variation of the second spherical harmonic term contributed by $E_{\mu \gamma}$ would not be easy to detect. Moreover the terms in $\sigma_{\mu \gamma}$ contribute with the desired sign to $X$ and, if $\sigma_{\mu \gamma}$ were close to the limit (eq. [77]) set by the cluster data, could contribute an appreciable part of the observed acceleration parameter. In any case, it seems premature to use the observed value of $h_{2}$ to distinguish between models until better information on angular variations is available. A first step would be to improve the estimate of equation (77).

For the area of a distant object we get the very simple form

$$
d A_{G}=r^{2} d \Omega_{0} .
$$

This relation holds in all theories, not just in general relativistic ones, and it is exact. Thus, any observed major deviation from equation (80) would be a catastrophe from the theoretician's viewpoint. Excluding this possibility, equation (80) can be combined with equations (68) and (70) in a variety of useful ways if $d A_{G}$ is more accurately known than $I_{G}, I_{0}$, or $f_{G} / f_{0}$. We then eliminate the least accurate quantity from these three equations to get a relation between observable quantities and the parameters of Table 1 . In fact, in practice it is often necessary to work with surface brightness rather than $I_{G}$ and then equation (80) is needed. Moreover, it may sometimes be possible to measure $d \Omega_{0}$ but not $f_{0}$. In the case of the Friedman models, for which the distortion vanishes, the apparent shape of the galaxy remains unchanged, and equation (80) then says that its apparent angular diameter is proportional to its proper linear diameter and inversely proportional to $r$. This is why McVittie calls $r$ ( $\xi$ in his notation) the distance by apparent size. If we use equation (75) to eliminate $r$ in favor of $z$, we obtain the usual angular-diameter-redshift relation (McVittie 1956, eq. [9.405]).

An interesting effect is the distortion. Let $i$ be a unit vector orthogonal to $e$, so that it is tangent to the observer's unit sphere. With $e$ fixed we let the Cartesicn components $i_{\mu}$ of $i$ vary over their one-parametric set of allowed values. We maximize the scalar

$$
i^{\mu} i^{\gamma} Z_{\mu \gamma}=i^{\mu} i^{\gamma}\left(E_{\mu \gamma}-2 e^{\lambda} \epsilon_{\lambda \mu \beta} H_{\beta \gamma}-e^{\lambda} e^{a} \epsilon_{\lambda \mu \beta} \epsilon_{\alpha \gamma \zeta} E_{\beta \zeta}\right)+O(r),
$$


where $\epsilon_{\mu \gamma \lambda}$ is the Levi-Civita symbol. The corresponding $i$ is the major axis of the elliptical image produced by an intrinsically spherical source. The ratio $e$ of major to minor axis is, from equation (38),

$$
e=1+\frac{1}{3} \max \left(i^{\mu} i^{\gamma} Z_{\mu \gamma}\right) r^{2}+O\left(r^{3}\right) .
$$

(Note that $e$ is not the magnitude of $e$.) If the source is not intrinsically spherical, its image will be distorted in the same proportion and in the same direction $i$. Thus, with the direction on the celestial sphere fixed, all distant objects will show an apparent statistical tendency to line up, and the effect is proportional to $r^{2}$. Suppose that at $r \approx 10^{9}$ years (that is, $z \approx 0.1$ ), we could still detect a distortion factor $e \approx 2$. Then, according to the estimate of equation (79), $E_{\mu \gamma}$ is too small to be detectable. But a detectable contribution from $H_{\mu \gamma}$ would occur if

$$
H_{\mu \gamma} \gtrsim 10^{-18} \text { year }^{-2} \text {. }
$$

The authors have tried unsuccessfully to find theoretical or observational evidence that such large values of $H_{\mu \gamma}$ cannot occur. They feel that a direct measurement of the distortion effect would be the best way to measure or exclude them. In terms of the redshift, equation (83) reads

$$
e-1=\max \left(i^{\mu} i^{\gamma} Z_{\mu \gamma}\right) z^{2} /\left(\frac{1}{3} \theta+e^{\lambda} e^{\beta} \sigma_{\lambda \beta}\right)^{2} .
$$

Note that the distortion vanishes for the Friedman models.

There are several favorable aspects of the distortion effect from the observational point of view. For $\boldsymbol{e}$ and $\boldsymbol{r}$ fixed, the distortion is the same for objects of any size and shape, so that all kinds of galaxies and even galactic groups could be included in the same statistics. It does not matter whether we see a whole galaxy or only its inner part. Even if absorption estimates are in error, this would affect only our numerical estimate of $H_{\mu \gamma}$, not the presence or absence of the effect. Finally it turns out that, although distortions can also be produced by the "lens effect" (light from the distant galaxy passing near another galaxy), these individual distortions are small and random, so that they do not mask the systematic cosmological effect. (We have used the estimate that light from a galaxy at $10^{9}$ years will not on the average come closer than $10^{4}$ years from the center of a second galaxy with a mass of $10^{10}$ solar masses.)

The distortion effect is also quite interesting from the theoretical point of view. In our other equations the curvature quantities $E_{\mu \gamma}$ and $H_{\mu \gamma}$ get mixed up with kinematical quantities; but only the curvature contributes to the distortion. However, the equations for the distortion effect do not depend on the assumption that general relativity is valid. Therefore, a positive result of such a measurement would merely indicate that space-time is curved (or that distant objects are systematically intrinsically lined up for some reason).

One additional possibility should be mentioned. It is conceivable that we happen to be in a region where $H_{\mu \gamma}$ is something like $10^{4}$ times as great as the estimate given in equation (83) but that within a distance of $10^{7}$ years the value of $H_{\mu \gamma}$ drops to zero or changes sign. Such a local fluctuation could be detected by analyzing the shapes of nearby objects, as in the work of Wyatt, Brown, and others (see Brown 1964 and the references given there).

The next relation of interest is the number of galaxies in solid angle $d \Omega_{0}$ between corrected luminosity distances $r$ and $r+d r$. If the intrinsic number density of sources is $K \rho$, where $K$ is a constant, then the number $d N$ of sources is, from equation (39),

$$
\begin{aligned}
d N & =K\left(f_{G} / f_{0}\right) r^{2} d r d \Omega_{0}\left\{\rho_{0}+r\left(\rho_{0} \theta+\rho_{\mu} e^{\mu}\right)+\frac{1}{2} r^{2}\left[\rho_{0}-\Lambda+\frac{4}{3} \theta^{2}+\sigma_{\mu \nu} \sigma_{\mu \nu}\right.\right. \\
& \left.\left.\left.-\omega_{\mu \nu} \omega_{\mu \nu}\right) \rho_{0}+2 e^{\mu}\left(\rho_{\nu} u_{\nu \mu}+\rho_{\mu} \theta+\rho_{0} u_{\nu \nu \mu}\right)+e^{\mu} e^{\nu} \rho_{\mu \nu}\right]+\ldots\right\} .
\end{aligned}
$$


If evolutionary effects are important, then the transition from equation (39) to equation (85) must be suitably modified. The observational evidence on number counts does not seem sufficiently clear at the moment for this relation to yield useful numerical estimates or upper bounds (Heckmann and Schücking 1959, 1962; McVittie 1956, 1959, 1962; Zwicky 1959). A very rough guess is $\rho_{\mu} \leq 10^{-8}$ year $^{-1}$ and $\rho_{\mu \nu} \lesssim 10^{-16}$ year $^{-2}$. In analyzing the data on the linear correction to the Newtonian result, attention should be paid to the possibility of a first spherical harmonic term; the second correction contains spherical harmonics of orders 0,1 , and 2 . In the actual observations it is often the indefinite integral of equation (85) over $d r$ which is found. The integration can be performed directly after substituting for $f_{G} / f_{0}$ from equation (70). If desired, $r$ can then be replaced by $z$ as independent variable by using equation (70) again. Finally, if the desired independent variable is $I_{0}$, one substitutes from equations (68) and (70) (after performing the integration) to eliminate $r$ and $z$. The resulting equations contain spherical harmonics of higher order than those which appear in equation (85). For the Friedman models, equations (69a), (69b), and (76) can be used to reduce (85) to the corresponding equation (9.304) of McVittie (1956).

Finally, we consider apparent proper motions. These are of interest mainly because they could provide an independent estimate or upper bound for the incident magnetictype field $H_{\mu \nu}$. If $e$ is kept fixed on a distant object its rate of change relative to a local gyroscope direction is, from equation (49),

$$
\begin{aligned}
& d e^{\mu} / d t=h^{\mu \nu}\left\{e^{\beta}\left(\sigma_{\nu \beta}+\omega_{\nu \beta}\right)+r\left[e^{\beta}\left(\sigma_{\gamma \beta}+\omega_{\gamma \beta}\right) u_{\nu \gamma}-e^{\beta} E_{\nu \beta}\right.\right. \\
& \left.\left.+\frac{1}{2} e^{\beta} e^{\gamma}\left(u_{\nu \beta \gamma}-\epsilon_{\nu \beta \lambda} H_{\gamma \lambda}\right)-e^{\beta} e^{\gamma} e^{\lambda}\left(\sigma_{\nu \gamma}+\omega_{\nu \gamma}\right) \sigma_{\beta \lambda}\right]+\ldots\right\} .
\end{aligned}
$$

Here $h^{\mu \nu}=\delta^{\mu \nu}-e^{\mu} e^{\nu}$. Suppose that we can measure an apparent proper motion of $1^{\prime \prime}$ per century. Then, according to our previous limits on $\sigma_{\beta \nu}$ and $\omega_{\beta \nu}$, the $r$-independent term in equation (86) is too small to be detectable. This means that, in practice, we can avoid the somewhat unrealistic appeal to a local gyroscope, since we can choose nearby galaxies as our standard of no proper motion to a high degree of accuracy. If a large number of nearby galaxies is used, then their peculiar motions will be averaged out. Thus, apparent proper motions of distant galaxies or groups relative to nearby ones would mean that the distant galaxies, not the nearby ones, have an apparent proper motion relative to a local gyroscope. Of course it may well be that the term in equation (86) that is linear in $r$ is also much too small to detect. Within the framework of this paper the point must be settled by observations, not by appeal to a philosophical argument that distant galaxies automatically show no proper motions.

Even if proper-motion studies could be made on objects at a distance of $10^{9}$ years, then the upper limit we could get for quantities not estimated from the redshift is not very good:

$$
H_{\nu \lambda,} u_{[\lambda \nu] \beta} \approx 10^{-16} \text { year }^{-2} .
$$

In particular, the upper limit placed by proper-motion measurements on $H_{\nu \mu}$ could probably never approach the accuracy of the distortion measurements (eq. [83]). However, the proper-motion measurements seem to be the only way to measure the part of $u_{\lambda \gamma \nu}$ that is antisymmetric in the first two indices. Note that the two terms estimated in equation (87) cannot be distinguished from each other by proper-motion observations; only the particular combination of them that enters into equation (86) can be measured by looking at $d e / d t$. The apparent proper motion vanishes in the Friedman models.

It is often claimed that the universe in the large must be isotropic or homogeneous. Certainly this view has immense aesthetic and philosophical appeal, but is it strongly supported by current observations? Unfortunately, it is not. We can get a rough idea of 
whether the anisotropy and inhomogeneity parameters have observational bounds that are "small" in the dynamical sense by using the inverse Hubble constant as a time scale. We use units for which $\frac{1}{3} \theta \equiv 1$. Examination of equation (70) or similar dynamical equations arising from higher coeffi ients in the expansion of equation (50) now yields a rough but simple criterion: quantities that are large compared to unity are dynamically important; quantities small compared to unity are not dynamically important.

Our upper bounds in these units are

$$
\begin{gathered}
\sigma_{\mu \gamma} \lesssim 0.5, \quad \omega_{\mu \gamma} \lesssim 1, \quad \Lambda, E_{\mu \gamma}, u_{(\mu \gamma \lambda)} \lesssim 2, \quad \rho_{0} \lesssim 10, \quad \rho_{\mu} \lesssim 10^{2}, \\
\rho_{\mu \gamma}, H_{\mu \gamma}, U_{[\mu \gamma] \lambda} \lesssim 10^{4} .
\end{gathered}
$$

Tentative as these numbers are, they show that, as yet, observations neither confirm nor deny the "cosmological principle" that the universe is isotropic and homogeneous, or even homogeneous, and that measurements at the present time cannot prove, but can only disprove, that particular models represent the actual structure of the universe.

In conclusion, it would be desirable to improve our considerations in several ways. There may be observable effects which we have not thought of. We can hope for more accurate numerical estimates from a careful study of existing data. And global theoretical models that are inhomogeneous should be looked for. As far as future measurements are concerned, it seems likely that an improvement of present accuracy by a factor between 10 and 100 might bring almost all the parameters in Table 1 within the range of observation. For $H_{\mu \nu}$, even an inspection of existing plates should yield a value or an improved upper bound. It may prove possible to improve our limit on $\sigma_{\mu \nu}$ by examining the angular dependence of the Hubble constant.

The idea of trying to analyze inhomogeneous models arose while the authors were attending an excellent series of lectures by Prof. G. de Vaucouleurs of the University of Texas. We are also grateful to Prof. E. Schücking of the Relativity Center for stimulating discussions on the theoretical aspects of this paper. A critical reading of the manuscript by Dr. R. H. Boyer was extremely helpful.

This research was supported in part by the Aerospace Research Laboratories, Office of Aerospace Research, and by the Office of Scientific Research (Grant 454-63), U.S. Air Force.

Note added in proof.-Some of the ideas and arguments in $\S$ II have been presented independently by David M. Zipoy ("Light Fluctuations Due to an Intergalactic Flux of Gravitational Waves" [preprint: University of Maryland Department of Physics and Astronomy, 1965]).

\section{REFERENCES}

Birkhoff, G. D. 1923, Relalivity and Modern Physics (Cambridge, Mass.: Harvard Univrsity Press), p. 124.

Bondi, H. 1947, M.N., 107, 410.

Brown, F. G. 1964, M.N., 127, 517.

Ehlers, J. 1961, Akad. Wiss. Mainz, 11, 804.

Heckmann, O., and Schücking, E. 1959, Encyclopedia of Physics, 53 (Berlin: Springer-Verlag), 489. Sons).

Hoyle, F. 1962, in Evidence for Gravitational Theories, ed. C. Mфller (New York: Academic Press), p. 141.

Hoyle, F., and Narlikar, J. V. 1961, Observatory, 81, 89.

Humason, M. L., Mayall, N. and Sandage, A. R. 1956, A.J., 61, 97.

Jordan, P., Ehlers, J., and Sachs, R. K. 1961, Akad. Wiss. Mainz, Vol. 1.

McVittie, G. C. 1956, General Relativity and Cosmology (London: Chapman and Hall). 1959, Encyclopedia of Physics, 53 (Berlin: Springer-Verlag), 445.

- (ed.). 1962, Problems of Extra-galactic Research (New York: Macmillan Co.), Part III. 
Murnaghan, F. D. 1938, The Theory of Group Representations (Baltimore: Johns Hopkins Press). Penrose, R. 1960, Ann. Phys., 10, 171.

Pirani, F. A. E. 1962a, in Recent Developments in General Relativity (New York: Pergamon Press).

1962b, article in Gravitation: An Introduction to Current Research, ed. L. Witten (New York: John Wiley \& Sons).

Sachs, R. K. 1961, Proc. R. Soc. London, A264, 309.

Sachs, R. K., and Bergmann, P. G. 1958, Phys. Rev., 112, 674.

Sandage, A. R. 1961, Ap. J., 133, 355.

359. 1962, Problems of Extra-galactic Research, ed. G. C. McVittie (New York: Macmillan Co.), p. 359.

Thomas, T. Y. 1934, The Differential Invariants of Generalized Spaces (Cambridge: Cambridge University Press).

Trautman, A. 1962, Recent Developments in General Relativity (New York: Pergamon Press), p. 459.

Zwicky, F. 1959, Encyclopedia of Physics, 53 (Berlin: Springer-Verlag), 390.

Open Access This article is distributed under the terms of the Creative Commons Attribution Noncommercial License which permits any noncommercial use, distribution, and reproduction in any medium, provided the original author(s) and source are credited. 